\title{
Téoros
}

Revue de recherche en tourisme

\section{Produits, destinations, particularités et enjeux de l'écotourisme en Europe}

\section{Sylvie Blangy et Françoise Kouchner}

Volume 21, numéro 3, automne 2002

Écotourisme

URI : https://id.erudit.org/iderudit/1072499ar

DOI : https://doi.org/10.7202/1072499ar

Aller au sommaire du numéro

Éditeur(s)

Université du Québec à Montréal

ISSN

0712-8657 (imprimé)

1923-2705 (numérique)

Découvrir la revue

Citer cet article

Blangy, S. \& Kouchner, F. (2002). Produits, destinations, particularités et enjeux de l'écotourisme en Europe. Téoros, 21(3), 20-27.

https://doi.org/10.7202/1072499ar d'utilisation que vous pouvez consulter en ligne. 


\section{Produits, destinations, particularités et enjeux de l'écotourisme en Europe}

\section{Sylvie Blangy et Françoise Kouchner}

\begin{abstract}
A pparue en Europe plus tardivement qu'en Amérique du Nord, la notion d'écotourisme a rapidement qualifié des concepts ou des projets fort variés, faisant référence au tourisme rural, naturaliste, alternatif, responsable, éthique, solidaire...
\end{abstract}

Le terme même d'écotourisme a été en grande partie supplanté par la notion de tourisme durable, notamment auprès de la Commission européenne (CE) et auprès des gouvernements. L'écotourisme est ainsi très rarement cité dans les documents administratifs, si ce n'est à l'occasion de l'Année internationale de l'écotourisme ou en citation des textes du PNUE (Programme des Nations Unies pour le développement) et de l'OMT (Organisation mondiale du tourisme).

Dans un tel contexte, la clarification des concepts opérée par l'OMT sur le tourisme durable et l'écotourisme s'est révélée opportune et a reçu un bon accueil de la part de l'ensemble des acteurs. L'Année internationale de l'écotourisme a permis aussi de faire émerger une réalité jusqu'alors peu identifiée.

\section{Spécificités et enjeux de l'écotourisme en Europe}

\section{Le rôle d'initiateur et d'aiguillon de la Commission européenne}

De 1995 à 2000, la Commission européenne a joué un rôle de leader dans le dé- veloppement du tourisme durable en Europe, grâce à des projets pilotes menés par les directions générales Environnement et Entreprise (Unité tourisme), les programmes LIFE (L'instrument financier pour l'environnement) ou d'autres outils de financement.

Parmi les nombreuses opérations mises en œuvre, quelques initiatives font figure de référence ${ }^{1}$ :

- le Grand prix du tourisme et de l'environnement pour les destinations (1995),

- la gestion intégrée de la qualité dans les stations rurales, urbaines et côtières (2000),

- la Charte européenne du tourisme durable dans les espaces protégés (1999),

- les lignes directrices du tourisme durable dans les sites Natura 2000 (2000).

D'autres programmes liés à des fonds spécifiques ont largement contribué au financement de projets territoriaux d'écotourisme, même s'ils ne sont pas identifiés comme tels ; le programme LEADER pour le tourisme en espace rural (Liaison entre action et développement de l'économie rurale), INTERREG (projets interrégionaux), FEDER (Fonds européen de développement rural) et les programmes de financement $\mathrm{PHARE}^{2}$ et TACIS pour les pays de l'Europe de l'Est et nouvellement indépendants.

Aujourd'hui la Commission européenne joue un rôle plus modeste, encourageant les pays membres à reprendre ces orientations à leur compte. Elle poursuit pour sa part son propre travail de réflexion de fond sur les questions de l'emploi, de la compétitivité et de la durabilité, réfléchissant notamment sur l'application de l'agenda 21 à tous les secteurs de l'industrie touristique et à l'évaluation de la qualité des services dans le tourisme. Quelques thématiques sont particulièrement explorées :

- l'amélioration de la qualité des produits, des prestations et des destinations touristiques,

- la protection de la nature et le développement durable (culture et transports),

- la gestion des répercussions des nouvelles technologies.

Parallèlement, la Direction générale Environnement soutient le programme VISIT (Volunteer Initiatives for Sustainability) qui vise la certification européenne des écolabels en tourisme, ainsi que leur promotion.

\section{L'écotourisme à l'européenne, une démarche originale et diversifiée}

Au-delà de son organisation institutionnelle, l'Europe est un continent densément peuplé, fortement anthropisé, aux paysages ruraux très diversifiés et où subsistent peu d'espaces naturels vierges et de communautés autochtones. L'Europe demeure par ailleurs marquée par une profonde disparité : quinze langues officielles, sans compter les langues régionales, des écosystèmes allant du boréal au désert et de profondes différences culturelles entre l'Europe du Nord et les pays de culture latine.

Aussi le terme d'écotourisme s'appliquerat-il à des espaces ou des contextes bien déterminés : aires protégées, sites de protec- 
tion des espèces ou habitats naturels (sites Natura 2000), territoires ruraux peu peuplés (tourisme rural), régions à forte diversité biologique (Europe de l'Est), régions où les populations autochtones vivent de la pratique d'une activité traditionnelle (éleveurs de rennes), initiatives de tour opérateurs (TO) européens dans les pays en développement...

L'écotourisme mis en œuvre en Europe présente des aspects extrêmement contrastés, marqués, bref, une prédominance des aspects « humanitaires » ou humanistes dans les pays dits latins ; la priorité est donnée aux projets de conservation et de préservation des espèces et des habitats chez les Anglo-Saxons.

\section{L'écotourisme, un savoir-faire et une ingénierie qui s'exporte à l'étranger}

Sur le plan international, l'écotourisme est devenu une thématique forte dans les programmes de coopération multilatérale ou bilatérale, en premier lieu en ce qui touche le marketing et la commercialisation des produits sur le marché émetteur européen. D'autres axes sont également considérés :

- Euro Aid - qui remplace au sein de la Commission européenne la Direction générale Développement - consacre des moyens importants aux projets intitulés « réduction de la pauvreté », « conservation de la biodiversité », « développement rural, local, communautaire »... avec des volets écotouristiques de plus en plus conséquents.

- À la suite de l'évaluation de ses projets, le Fonds Français pour l'Environnement Mondial (FFEM) utilise désormais la démarche d'écotourisme comme un outil de valorisation de la biodiversité et du développement communautaire.

- Par ailleurs, en France, le Ministère des Affaires étrangères a décidé d'investir 1,2 million d'euros dans un projet d'aide à la coopération par le tourisme pour 2002-2005. L'objectif est d'élaborer des modes de développement touristique qui font participer les populations concernées, valorisent les patrimoines naturels et culturels des territoires et génèrent des flux financiers dirigés vers la gestion des ressources et des projets de développement durable.

\section{L'Année internationale de l'écotourisme et ses suites}

Quatre conférences thématiques régionales ont eu lieu, soit en Europe, soit concernant le territoire européen :

- les régions montagneuses, les transports et la mobilité douce (Autriche);

- les îles et les régions méditerranéennes (Grèce);

- les régions arctiques, y compris le Nord de la Russie et le continent nord-américain (Suède);

- les régions désertiques, dont l'Afrique du Nord (Algérie).

Si les conférences régionales ont mis au jour le discours ambigu de l'Europe, évoquant d'abord et surtout le tourisme durable, elles ont été le lieu de riches débats, notamment concernant les définitions appropriées de l'écotourisme, et elles ont créé de véritables dynamiques.

Les rencontres ont permis un nouveau regard de l'Europe sur ses marges. De nouvelles destinations comme la Sibérie ont pu se faire connaître et présenter des expériences méconnues jusqu'alors du public européen ; des mouvements de solidarité avec des destinations défavorisées par leur contexte politique (désert algérien) sont apparus. Les régions périphériques à l'Europe ont confirmé leur intérêt pour le marché européen de l'écotourisme et demandé à ce que les partenariats avec les tour opérateurs et les collectivités territoriales (coopération décentralisée) soient renforcés, en particulier dans la phase de conception et de mise en marché des produits. Elles ont révélé par ailleurs la nécessité pour les tour opérateurs de se faire mieux identifier comme opérateurs responsables et de mieux organiser leurs partenariats avec les prestataires d'écotourisme des pays récepteurs, en Europe ou hors de l'Europe.

De façon générale, l'Année internationale de l'écotourisme a impulsé des évolutions apparemment de fond, l'écotourisme de- venant par exemple le fil conducteur de projets menés à une échelle territoriale (les parcs naturels régionaux) ou suscitant une demande accrue de formation. Le terme écotourisme a ainsi fait son entrée dans le langage officiel...

D'autres rencontres suivent ou vont suivre les conférences régionales : Sofia en Bulgarie (octobre 2002), IIPT à Genève (Institut international pour la paix par le tourisme, février 2003), Hanovre, Forum Initiative (janvier 2003), sans oublier toute une série d'ateliers et de rencontres aux thématiques pointues.

De cette mise en marche, de ces brassages d'idées, émergent clairement des axes de travail déterminants pour le proche avenir de l'écotourisme en Europe :

- une meilleure connaissance des marchés européens,

- un développement de produits innovants basés sur le partenariat entre clients, voyagistes et prestataires,

- des outils marketing qui facilitent la mise en marché des prestataires,

- un appui à l'organisation des territoires d'accueil,

- la promotion des labels et leur certification,

- une plus grande coopération entre pays émetteurs et récepteurs.

\section{Mieux connaître le marché de l'écotourisme en Europe}

\section{Des études de marché à compléter}

Il n'existe pas réellement d'étude sur le marché de l'écotourisme à l'échelle européenne. Comblant en partie cette lacune, l'OMT (2002) a commandité des études sur les cinq principaux marchés émetteurs et récepteurs de tourisme en Europe : l'Italie, la France, l'Allemagne, l'Espagne, le Royaume-Uni. La Suisse et l'Autriche ont ensuite produit leurs propres études en suivant le cadre établi par l'OMT. Les études de l'OMT seront complétées par la présentation d'analyses plus récentes au $1^{\mathrm{er}}$ Salon européen de l'Écotourisme à Hanovre, fin janvier 2003 (GTZ, 2002). 
Difficilement comparables, malgré une méthodologie et des questionnaires similaires, les études livrent, pour chaque pays, les grandes tendances ainsi que les principales caractéristiques du marché européen :

- le marché de l'écotourisme est un segment en pleine expansion dans tous les pays ;

- l'Europe est le plus gros marché émetteur d'écotourisme dans le monde, ainsi que le plus accessible ;

- les tour opérateurs jouent un rôle moteur dans la construction du produit et constituent un groupe d'interlocuteurs privilégiés pour les prestataires des pays récepteurs ;

- les voyages éloignés devraient continuer d'augmenter ;

- parallèlement, les destinations européennes vont se développer en fonction de leur capacité à valoriser leur potentiel et à s'organiser (marché national, Méditerranée, Maghreb et Europe de l'Est).

\section{L'Europe - le plus gros marché d'écotourisme dans le monde}

Principal marché récepteur et émetteur de tourisme dans le monde, l'Europe est aussi le plus gros marché émetteur d'écotourisme (tableau 1). Sur le marché récepteur, en 1999, l'Union européenne (UE) représentait $43 \%$ des arrivées et $40 \%$ des recettes du tourisme mondial. Les cinq pays étudiés par 1'OMT représentent à eux seuls $25,75 \%$ des arrivées de touristes à l'échelle internationale.

La France (60 millions d'habitants) vient en tête des pays récepteurs avec 75 millions de touristes en 2001 et 95 millions prévus en 2011. L'Espagne est la troisième destination mondiale avec 48,5 millions de visiteurs, derrière les États-Unis. L'Allemagne, puis le Royaume-Uni, représentent quant à eux de puissants marchés émetteurs.

Les cinq études de l'OMT mentionnées évaluent que le volume du marché de l'écotourisme représente de 5 à $20 \%$ du marché actuel et qu'il vivrait une crois- sance trois fois supérieure à celle du tourisme classique (Ecosystems, 2002).

En pleine expansion, le marché européen d'écotourisme apparaît moins affecté par les aléas économiques et politiques des pays récepteurs et plus diversifié en termes de socio-styles, de demandes et de pratiques que le marché nord-américain. Rien d'étonnant qu'il soit convoité par plusieurs pays récepteurs d'Amérique latine, le Brésil en particulier, qui mobilisent des moyens importants pour s'y positionner.

\section{Les destinations d'écotourisme : l'étranger et la proximité tout à la fois}

Les Européens voyagent partout dans le monde et peu de destinations sont considérées comme purement écotouristiques. Les préférences vont aux îles, aux régions tropicales et aux pays avec lesquels chaque pays émetteur entretient des relations privilégiées (anciennes colonies, protectorats, communautés de langue...).

Les destinations européennes (tableau 2), les pays limitrophes de l'Europe et le pourtour méditerranéen présentent pour leur part un grand potentiel pour l'écotourisme et font l'objet d'une demande croissante, notamment depuis le 11 septembre 2001. De nombreux facteurs pèsent « durablement » en leur faveur : l'existence de la monnaie unique (l'euro), la réduction du temps de travail, un souci accru de sécurité, la prise de conscience de l'impact des transports aériens.

Pays tout à la fois émetteurs et récepteurs, la France, l'Espagne et l'Italie renforcent leur rôle de destination d'écotourisme pour les clientèles du Nord. L'attraction pour le pourtour méditerranéen demeurant très marqué, l'Espagne a pris des mesures pour améliorer la qualité des offres insulaires (Canaries, Baléares). Les pays du Maghreb et du Nord de l'Afrique ont également leur place dans cette offre méditerranéenne à condition qu'ils sachent se réorganiser. Le Maroc prend les devants et lance un programme national de tourisme durable avec l'aide de la coopération américaine (USAID).

\begin{tabular}{|l|c|}
\hline \multicolumn{2}{|c|}{ L'Europe comme marché émetteur } \\
\hline Pays & Nombre de départs en 1999 (milliers) \\
\hline Allemagne & 73400 \\
\hline Royaume-Uni & 53881 \\
\hline Italie & 18962 \\
\hline France & 16709 \\
\hline Espagne & 4794 \\
\hline TOTAL & 167746 \\
\hline Source: OMT & \\
\hline
\end{tabular}

\begin{tabular}{|l|l|}
\hline \multicolumn{2}{|c|}{ Les destinations préférées des Européens } \\
\hline Espagnols & Amérique latine (Brésil, Argentine) \\
\hline Italiens & Amérique latine \\
\hline Français & $\begin{array}{l}\text { Afrique du Nord et Afrique francophone, } \\
\text { Maroc }\end{array}$ \\
\hline Britanniques & Népal, Pérou et Équateur, \\
\hline Allemands & $\begin{array}{l}\text { Europe (Espagne), Costa Rica, } \\
\text { Canada et Équateur }\end{array}$ \\
\hline
\end{tabular}

Par ailleurs, les pays de l'Europe de l'Est s'emploient à se positionner rapidement sur le marché de l'écotourisme de l'Europe occidentale, avec des offres appropriées. Destinations tout à la fois proches et exotiques, rassurantes car culturellement moins éloignées, offrant une grande diversité faunistique et des cultures traditionnelles encore fortes, ces régions répondent aux nouvelles attentes.

\section{Le rôle pionnier des tour opérateurs}

\section{Une nouvelle génération de voyagistes responsables}

Les séjours d'écotourisme organisés à l'étranger représentent une offre diversifiée et originale, quoique encore limitée en nombre de départs. Cette offre est portée par une poignée de tour opérateurs, véritables pionniers, voire militants en écotou- 
risme. Ces opérateurs relèvent des secteurs privé et associatif, spécialistes de trek ou de voyages naturalistes, ONG (organisations non gouvernementales) de développement. Leur nombre varie de 20 à 40 tour opérateurs par pays.

La première étape de leur engagement a été de concevoir des codes de comportement et de prodiguer des conseils aux voyageurs, les incitant à avoir une démarche responsable. Leurs documents ont servi de ligne de conduite et de cadre contractuel avec les prestataires locaux dans les pays visités.

Ces tour opérateurs ont peu à peu éprouvé le besoin de se regrouper, de s'associer dans la réalisation de projets communs leur permettant de mieux se faire identifier et valoriser par rapport au reste du secteur des voyagistes.

\section{Se regrouper pour progresser}

Souvent citée comme exemple de groupement de tourisme durable et alternatif, Forum Anders Reisen, une association de 80 TO allemands, en plus de s'engager dans une Charte des voyagistes, finance en commun un site Internet, un stand collectif dans les salons du tourisme (Reisepavillon), des projets de développement dans les pays visités.

En Grande-Bretagne, l'Association des tour opérateurs indépendants (AITO Association of Independent Tour Operators - www.aito.co.uk), réunit 150 entreprises autour de lignes directrices pour un tourisme responsable.

En France, un groupe informel d'une dizaine de voyagistes spécialisés dans le trek et les déserts s'est constitué pour publier la charte Déserts Propres et Respect du Désert, lancer une campagne de nettoyage des sites de camping du Sud de l'Algérie et contribuer à la formation des guides locaux.

D'autres association de TO responsables transcendent les frontières : IATO (International Association of Antarctic Tour Operators) regroupe par exemple des TO qui travaillent sur l'Antarctique. TOI -

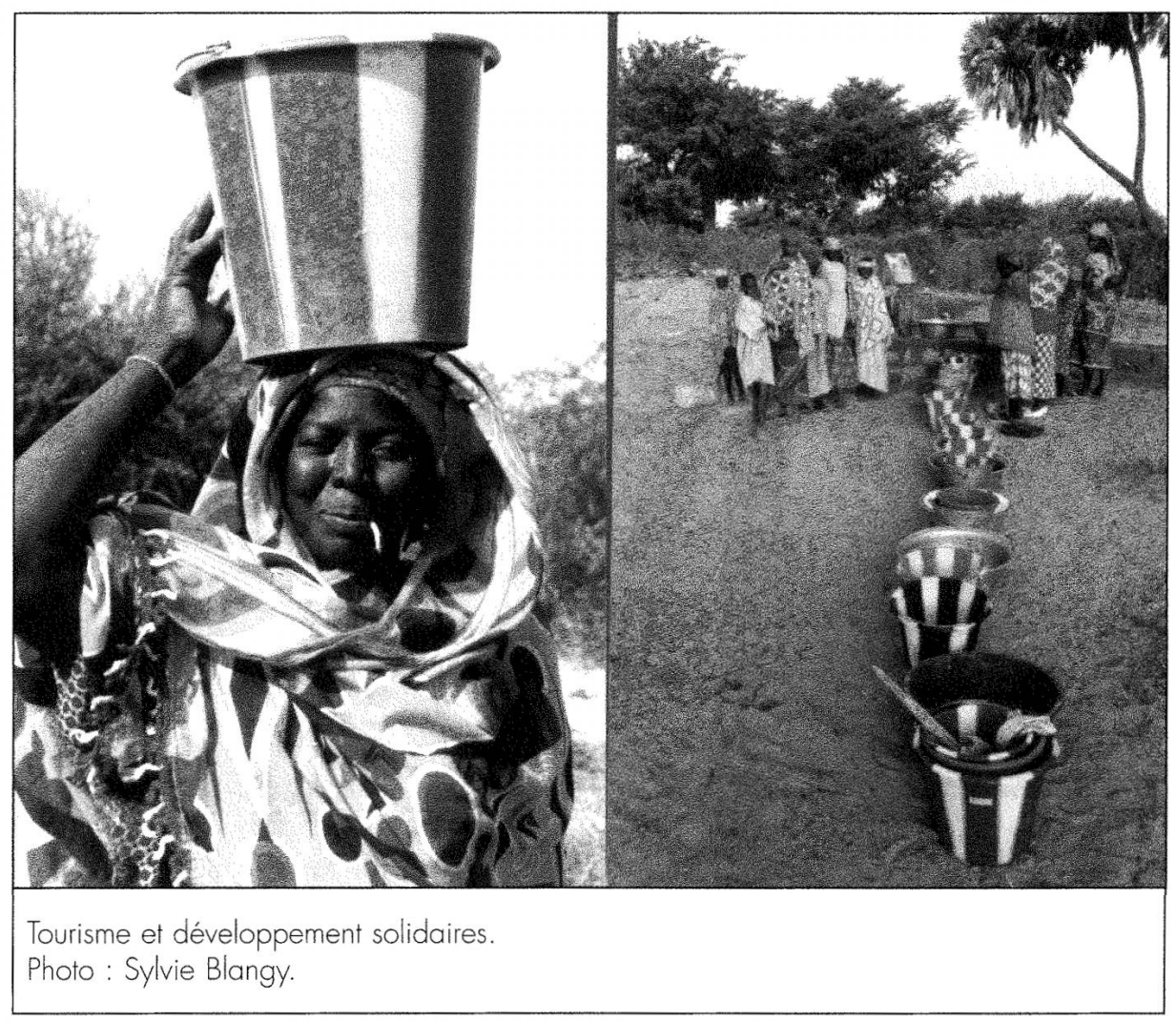

Tour Operator Initiative (www.toiniative. org), s'appuie sur 24 tour opérateurs de grande envergure, comme TUI (Thomson Travel Group www.tui-environnement. com) en Allemagne, qui jouent le rôle de grand frère et expérimentent un programme d'action et une stratégie de tourisme durable qu' on leur demandera de reproduire et de diffuser dans leur propre pays. Chaque membre de TOI se doit d'intervenir au nom de TOI et de faire participer l'ensemble du réseau à des actions partenariales avec une destination. C'est ainsi que les membres de TOI sont intervenus dans une des régions les plus touristiques de Turquie.

\section{De l'écotourisme au tourisme équitable et solidaire}

Cet important travail à l'étranger est relayé et amplifié par des ONG de développement. Le terme d'écotourisme cède alors le pas au tourisme dit « équitable», « responsable » ou « solidaire », glissement sémantique qui souligne la dimension résolument sociale, voire politique, de la plupart des initiatives.
Le débat est actuellement très ouvert sur le contenu du tourisme équitable et sur la labellisation éventuelle des produits s'affichant dans cette démarche. Sur le plan international, une réflexion menée par Tourism Concern (www.tourismconcern. org.uk) est conduite pour une mise en réseau des opérateurs et des prestataires locaux dans l'association Fair Tourism Network.

\section{Une nouvelle génération de produits pour un tourisme participatif et solidaire}

Ces engagements volontaristes se sont traduits par une production originale. Certaines structures commerciales ont consacré l'ensemble de leur production à des produits tournés vers le développement solidaire. Encore limitée en volume, cette nouvelle génération de produits écotouristiques est inondée par l'évolution générale des produits d'aventure et de trek vers une éthique et des responsabilités accrues. À l'origine plutôt voués à l'observation de la faune ou de sites naturels et culturels, de nombreux produits d'écotourisme deviennent également participatifs, souvent identifiés comme tels sous 
l'appellation de produits « écosolidaires », « écovolontaires ».

\section{Brève typologie}

\section{Séjours basés sur la rencontre ou l'immersion culturelle}

La seule présence de visiteurs peut contribuer au développement de villages et de campements éloignés des grands centres touristiques.

\section{...s'immerger dans une communauté villageoise au Burkina Faso}

Le concept de Village d'Accueil mis au point par l'association Tourisme et Développement Solidaire s'inspire directement des modèles de l'agritourisme et du tourisme social, adaptés aux communautés rurales des pays du Sud.

Une communauté villageoise labellisée Village d'Accueil est en mesure d'organiser des séjours d'une semaine basés sur la découverte du milieu, l'artisanat, la musique, les rencontres culturelles, etc. pour de petits groupes d'une douzaine de voyageurs. L'animation et l'hébergement sont assurés par une vingtaine de villageois recrutés par la communauté qui gère directement l'activité. Les bénéfices générés sont réinvestis dans des programmes de développement appuyés par des organismes locaux.

\section{Séjours de la connaissance}

«Voyager pour connaître, connaître pour aimer, aimer pour respecter. » Découvrir et apprendre peuvent se faire dans une démarche de soutien et de protection des espèces observées, le voyagiste ayant tissé des liens avec des missions scientifiques de conservation et de protection de la nature.

\section{Des séjours d'écovolontariat}

Le voyagiste propose des séjours « écosolidaires », où les volontaires participent financièrement à un programme de préservation : protection des loups, des ours et des lynx en Europe de l'Est, chez les Amérindiens, en Amérique latine.

\section{Contribution financière à des projets de développement local}

L'association de voyage prélève un pourcentage de ses recettes pour financer des actions de développement gérées par une ONG locale créée dans ce but. Chargée de la mise en place et du suivi des projets de développement, l'association locale intervient dans les domaines de l'eau, de la santé et de l'éducation et travaille en partenariat avec les associations locales.

Le partenariat avec l'Association Echagill, campement touareg au Mali

Au Mali, Croq'Nature est un partenaire du campement chamelier d'Echag (530 personnes) qui organise des randonnées chapelières. Réunis autour du chef de tribu et de ses conseillers, une association, au fonctionnement très démocratique, veille à ce qu'un maximum de nomades bénéficie des retombées touristiques.

Plusieurs classes ont été créées avec les recettes de la saison, en plus d'un point santé et d'un local coopératif artisanal géré par les femmes.

Pour gérer les projets de développement, le voyagiste peut décider de créer une fondation au sein même de son entreprise ou de s'appuyer sur une structure existante. Le projet Écol-liées financé par la fondation Allibert fait par exemple le lien, par le biais des groupes d'adeptes de trekking, entre les écoles andines des hauts plateaux et les écoles françaises.

\section{Création de conditions plus favorables aux populations locales}

Les conditions de production des voyages ayant souvent une incidence lourde sur les conditions de vie de la population, des voyagistes proposent des séjours dont les conditions sont plus favorables aux populations locales, en répercutant sur le prix du voyage les surcoûts occasionnés. Les séjours concernés, dont le prix est légèrement augmenté, sont alors signalés dans le catalogue par un pictogramme.

Une évaluation des produits participatifs reste à faire, notamment en ce qui concer- ne le nombre de projets réalisés, le taux de satisfaction du client, les retombées sur la communauté locale...

\section{En Europe, valoriser les terroirs et les cultures autochtones}

L'offre européenne d'écotourisme sur le territoire européen existe, se développe, se structure. Peu visible car diffuse, dispersée, peu identifiée comme telle, l'offre d'écotourisme s'est forgée sur différentes voies : développement des hébergements en espaces ruraux, valorisation du patrimoine, valorisation des produits du terroir, essor des loisirs de pleine nature.

$\mathrm{Si}$, pour une grande partie de cette offre, le touriste compose lui-même son séjour de façon autonome, à partir de prestations individualisées, une nouvelle génération de produits voit le jour, issue d'un partenariat entre les gestionnaires d'un territoire et les tour opérateurs nationaux.

\section{Écotourisme en Europe}

En Laponie, au Nord de la Scandinavie, les Samis, communauté autochtone vivant de l'élevage traditionnel du renne, s'organisent pour accueillir des visiteurs dans des köta en tourbe ou des tentes d'été et offrir des produits d'itinérance avec des rennes de bât. Leur culture et leur savoir-faire traditionnel (marquage des rennes, transformation des peaux et des cornes en produits d'artisanat) forment le cœur du produit touristique sous la forme de partage des activités quotidiennes.

En Belarus, dans la réserve de Bérézinski, les séjours naturalistes commercialisés par les tour opérateurs anglais et français permettent de partir sur les traces des grands mammifères (ours, loups, bisons) et de découvrir la vie traditionnelle dans les villages périphériques à la réserve mondiale de la biosphère, basée sur l'agriculture, la cueillette en forêt et la chasse.

\section{Faciliter la rencontre entre l'offre et la demande}

La rencontre entre les offres de produits d'écotourisme et les clients est encore dif- 
ficile et aléatoire : les volumes de clientèle traités sont faibles, les clients recherchent une information détaillée et des prestations de plus en plus personnalisées avec un contenu parfois très élaboré.

C'est la raison pour laquelle on assiste à de nombreuses initiatives qui visent à faciliter la rencontre entre les voyagistes spécialisés et les communautés autochtones ou les prestataires, souvent organisés en toutes petites structures.

\section{Impulser le partenariat entre voyagistes et destinations}

En amont, sur le lieu de construction du produit, le Testour, nouvelle version du traditionnel Eductour, fait participer les tour opérateurs en les transformant en partenaires actifs.

Sélectionnés pour leur expérience de partenariat avec des parcs ou des communautés autochtones, les tour opérateurs sont invités, dès le démarrage d'un projet, à participer au montage des produits, à vérifier les prestations existantes, à proposer des modes de partenariat et à s'impliquer comme ambassadeurs de la destination. Le parc du Pantanal au Brésil, créé sur le modèle des parcs naturels régionaux français et l'association des Pousadas Pantaneiras (APPAN) a mis en place un véritable contrat d'engagement avec huit tour opérateurs d'Europe et d'Amérique du Nord invités dans un Fam Tour (familiarisation) soutenu par la VARIG, compagnie aérienne brésilienne.

En aval, les prestataires des régions éloignées sont invités à présenter leurs produits à des foires et à des salons spécialisés, comme Reisepavillon en Allemagne.

\section{Reisepavillon, salon allemand et européen du tourisme alternatif et responsable}

Organisé à Hanovre, le Reisepavillon a été choisi en 2002 par l'OMT et le PNUE pour lancer officiellement l'Année internationale de l'écotourisme. Les organisateurs et la coopération allemande ont invité les ONG de développement, les associations villa-

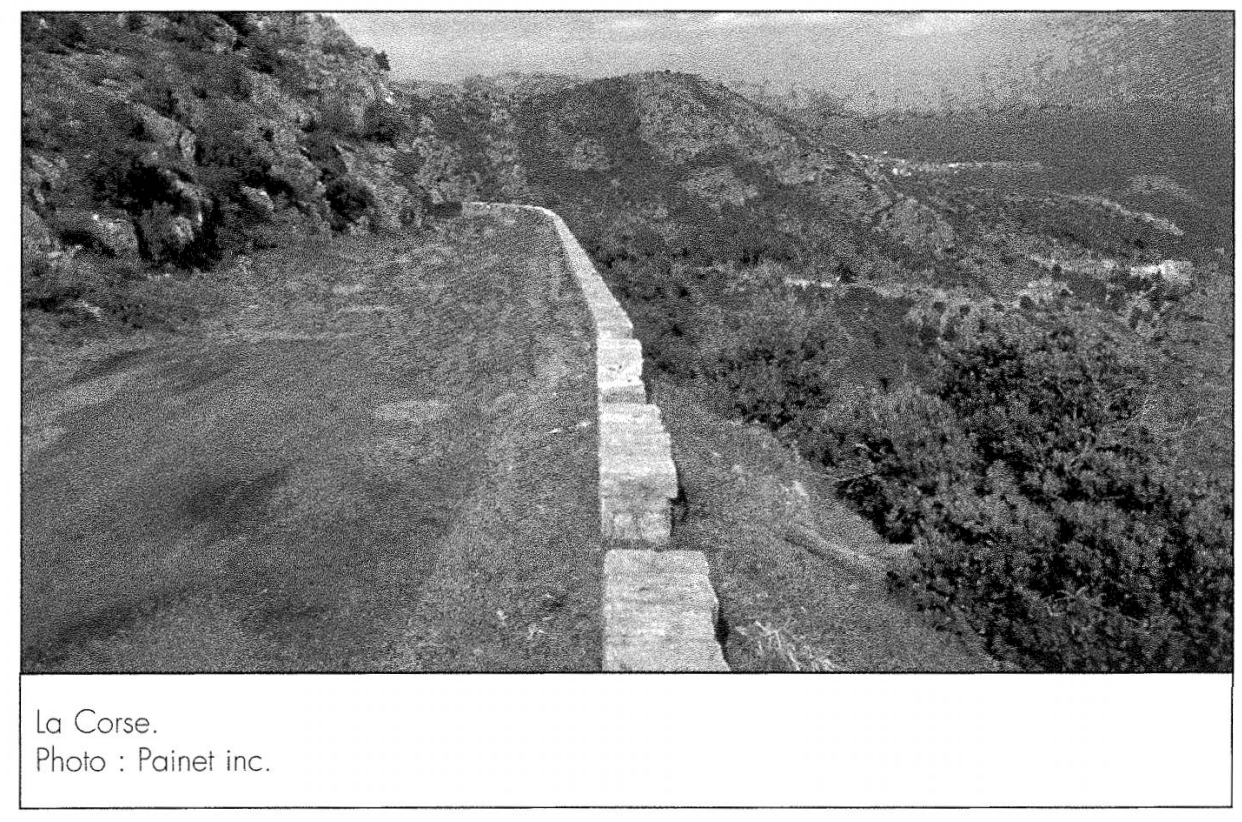

geoises et les communautés autochtones à présenter leurs séjours de tourisme communautaire. Les acheteurs étaient principalement des TO allemands regroupés entre autres dans l'association Forum Anders Reisen. Soixante-dix TO de tourisme responsable y étaient représentés.

Cette rencontre entre marché récepteur et marché émetteur allemand a été une telle réussite qu'elle sera reconduite en 2003 avec une dimension européenne, grâce à la participation active de six agences de développement de pays européens. Ces dernières profiteront du Forum de Hanovre pour définir une stratégie commune, en particulier en ce qui regarde les aspects promotion et marketing en Europe des projets qui génèrent des produits d'écotourisme. Un service de mise en relation entre prestataires locaux et tour opérateurs sera expérimenté pour la première fois par l'ECEAT (European Centre for Eco and Agro Tourism) dans le cadre du programme VISIT (programme visant la certification européenne, voir infra).

\section{Une préférence pour les salons spécialisés, régionaux et thématiques}

De façon générale, le marketing de l'écotourisme donne une nette priorité aux réseaux spécialisés (salons, presse, médias). Véritables vitrines des produits nature et découverte, les salons régionaux et thé- matiques se multiplient et prennent une ampleur grandissante. Ainsi, le salon de la randonnée de Paris a gagné en nombre d'exposants et de visiteurs au détriment du Salon mondial du tourisme organisé simultanément. Dans les grands salons, la tendance est au regroupement qui permet d'être mieux identifié par les visiteurs.

\section{Internet, un outil marketing privilégié pour l'écotourisme}

L'écotourisme fait la part belle à Internet et à ses déclinaisons : sites individuels, sites collectifs regroupant les prestataires sur une destination ou les voyagistes autour de principes de base...

Déjà très présents, les sites Internet des tour opérateurs sont évidemment destinés à se développer encore. Au-delà de la rapidité et de la qualité de l'information fournie aux clients et des possibilités de commercialisation aisée, Internet permet une interaction très appréciée : livre d'or consultable, carnets de route, bulletins d'information électroniques, forums de discussion... Les sites Internet détrônent petit à petit les brochures par la qualité des photos et la mise en scène des produits.

Internet pour évaluer,

labelliser et promouvoir

De nombreux sites Internet, financés dans le cadre de projets européens, mettent en 
ligne des produits, des projets ou de l'information sur le tourisme durable ou l'écotourisme.

Les offres de produits proposées dans le site ECO-TOUR (www.eco-tour.org) géré par The Friends of Nature International (www.nfi.at) en Autriche sont évaluées en fonction d'une grille de critères et réactualisées régulièrement.

ECO-TIP (www.eco-tip.org) est une initiative d'Ecotrans (www.ecotrans.org) basé en Allemagne; il liste toutes les initiatives de tourisme durable en Europe, en particulier les Écolabels, les récompenses, les cent meilleures pratiques et un forum de discussion sur la pertinence des Écolabels en Europe.

Le site anglais Responsible Travel (www.responsibletravel.com) fournit de l'information sur le tourisme en communautés locales et les tour opérateurs membres du réseau.

VISIT (Volunteer Initiatives for Sustainability) : finalement, dans le cadre du programme de promotion des dix meilleurs écolabels en Europe et de la campagne «Your visit makes the difference » (www.yourvisit.info), le site Internet renvoie aux principaux projets et initiatives mentionnés précédemment et prépare le terrain pour la future campagne de marketing électronique au service du tourisme durable nommée le Virtual Green Travel Market Center (www.greentravel market.info). Un site spécifique sera ouvert en janvier 2003 à l'occasion du premier salon de l'écotourisme en Europe (cf. Reisepavillon et Forum Initiative).

Les classiques outils marketing du tourisme de masse ne sont pas négligés, en premier lieu les traditionnels produits d'édition : l'ECEAT (European Centre for Eco Agro Tourism) édite le catalogue Réseau de fermes d'accueil écologiques, la Fédération des parcs naturels régionaux répertorie ses « Vacances au naturel » dans une brochure annuelle, les gites Panda sont connus en grande partie grâce à leur catalogue.

\section{Améliorer et garantir la qualité des prestations}

La certification et la labellisation sont devenues rapidement des outils privilégiés de la démarche écotouristique. Ainsi, $41 \%$ des Allemands donnent une préférence aux hébergements soucieux d'environnement et $19 \%$ recherchent des produits certifiés dans les catalogues de voyage.

Afin de garantir la qualité des prestations offertes et d'inciter les prestataires à améliorer leurs services, on a vu proliférer des opérations de labellisation partout en Europe : plus de quarante initiatives de labellisation ont été recensées et analysées dans l'étude de l'OMT conduite par Ecotrans (Ecotrans, 2002), les hébergements étant les plus nombreux à faire l'objet de procédure de labellisation.

Un premier bilan de ces initiatives apporte un constat unanime : diversité, richesse, mais aussi foisonnement, dispersion. La confusion règne, les labels sont peu visibles pour le consommateur et demeurent peu efficients dans le choix de la destination ou de la prestation.

Trop de labels tuant le label, l'heure est maintenant à l'évaluation, voire à la labellisation des labels et à leur réorganisation... La Direction générale Environnement de la $\mathrm{CE}$ a décidé de financer un vaste programme de promotion des écolabels en Europe avec les objectifs suivants :

- promouvoir et valoriser les meilleurs écolabels en Europe;

- faciliter la commercialisation des produits et des prestations labellisés;

- développer des indicateurs communs;

- mettre en place un programme de certification européen; certifier les meilleures opérations de certification.

C'est ainsi qu'est né le programme VISIT. Les dix labels européens les plus représentatifs ont été regroupés dans une plateforme de réflexion.

Clef verte, Gites Panda-

des labels reconnus

- Parmi les dix labels pionniers reconnus en Europe, on peut citer Clef Verte qui qualifie campings et caravanings et qui est attribué sur la base de 129 critères portant essentiellement sur les économies d'énergie, le tri, les énergies renouvelables, la gestion de l'eau, la sensibilisation à l'environnement. En France, 49 camping ont été labellisés en 2001.

- Dans la même démarche, le label gîte Panda est géré conjointement par le Fonds mondial pour la nature France (WWF - World Wildlife Fund), la Fédération des Parcs naturels régionaux de France et la Fédération des Gîtes de France. Les principaux critères de labellisation touchent la qualité de l'environnement, du gîte et sa gestion :

- offrir la possibilité de pratiquer l'observation de la nature dans un environnement naturel de qualité;

- comporter un équipement d'observation et d'information (malle pédagogique);

- faire une gestion de l'établissement soucieuse de la préservation de l'environnement, bien que les critères précis à ce sujet manquent;

- se trouver à proximité de départs ou de passages de chemins de randonnée.

Le gestionnaire du Gîte Panda s'engage à préserver les richesses naturelles remarquables autour de sa propriété. Actuellement au nombre de 258, les gîtes Panda sont quasiment tous implantés sur le territoire des pares régionaux ou nationaux. Ils connaissent un succès avéré auprès des clientèles étrangères et affichent un excellent taux de remplissage.

- Après plusieurs années de concertation avec les professionnels, le Parc national de la Guadeloupe (France) a décidé de créer en 1998 une marque de confiance intitulée « Recommandé par le Parc national de la Guadeloupe ». La marque peut s'appliquer à des gîtes, à des activités de pleine nature et à des sites aménagés (parcs floraux, jardins, musées). Les réalisations labellisées reprennent à la lettre les fondements de l'écotourisme quant au respect de l'environnement, à la sensibilisation des visiteurs et à la valorisation des patrimoines naturels et culturels. 
- En 2002, trente-trois produits se sont vus accorder la marque de confiance du Parc national de la Guadeloupe (écolodges, gîte créole, VTT des mers, maison du café, du cacao) et les prestataires concernés se sont regroupés dans l'Association Guadeloupéenne d'Écotourisme (AGE -www.ecotourisme.org).

La démarche intégrée d'écotourisme du parc naturel du Vercors

- Candidat à la Charte européenne du tourisme durable, le parc naturel régional du Vercors (France) mène depuis dix ans un vaste programme d'organisation de l'activité touristique conjuguant les grands principes du tourisme durable et de l'écotourisme :

- élaboration d'un schéma d'aménagement des activités sportives et de pleine nature, intégrant les retombées économiques et les impacts environnementaux (étude d'impact sur les nouveaux sites) ainsi que la gestion des conflits d'usage, sur les sites de canyoning en particulier ;

- création d'une offre spécifique de tourisme durable basée sur les gîtes Panda, les Hôtels au Naturel et les produits agro-bio, à la suite d'un éductour avec les tour opérateurs nord-européens ;

- création d'une marque «parc Vercors» avec un référentiel qui lui est propre, attribuée dans un premier temps aux produits agricoles (viande, truite), puis étendue aux hébergements (gîtes, centre de vacances) ;

- gestion environnementale renforcée mettant l'accent sur les critères environnementaux exigés par les Gîtes Panda et les Hôtels au Naturel ;

- entente avec l'Agence nationale de développement de l'énergie (ADEME) pour le développement des énergies renouvelables ;

- sensibilisation des résidants et des visiteurs lors des sorties estivales de découvertes du Parc et des cycles de conférences annuelles sur le patrimoine.

\section{Vers la poursuite du développement de l'écotourisme}

Le marché européen de l'écotourisme est destiné à s'affirmer grâce à la convergen- ce de deux dynamiques distinctes : le développement du marché de niche du tourisme équitable, solidaire et responsable, ou naturaliste, et l'évolution d'une partie du grand public, amenée à d'autres pratiques touristiques lors de séjours à l'étranger et goûtant, en Europe même, aux plaisirs d'une redécouverte intelligente du territoire.

\section{Sylvie Blangy est une consultante} française spécialisée en écotourisme. Son expertise touche autant la certification, le développement de produits, l'analyse de marché, la planification nationale ou régionale que les démarches de conservation de la biodiversité.

Françoise Kouchner est une consultante française spécialisée en tourisme de nature. Elle a notamment rédigé ou corédigé plusieurs rapports et guides de savoirs-faire publiés par l'Agence Française d'Ingenierie Touristique, sur la randonnée, le vélo tout terrain et l'écotourisme.

\section{Notes}

1 L'ensemble des publications issues des opérations initiées par l'Europe sont disponibles sur le site Internet de la CE.

2 Programmes destinés à assister les pays dans la reconstruction de leur économie.

3 Cf. infra.

\section{Bibliographie}

Blangy, S., et S. Vautier (2001), « Ecotourism in Europe », dans David B. Weaver (ed.), The Encyclopedia of Ecotourism, CABI Publishing. Blangy, S. (2000), « Lignes directrices et chartes du tourisme durable dans les espaces protégés en Europe. Analyse des initiatives existantes. Bonnes pratiques », Actes du colloque de Lisbonne, Les cahiers d'Espaces, numéro sur le Tourisme durable.

Blangy, S., G. Dubois, et F. Kouchner (2002), «Écotourisme, Expériences françaises - AFIT - Agence Française de l'Ingénierie Touristique », Les Cahiers de l'AFIT - Guide de Savoir-Faire.
Blangy, Sylvie, Ghislain Dubois, et Françoise Kouchner (2202), Ecotourism, The French Experience, AFIT collection, Paris.

Blangy, S., R. Denmann, et H. Hamele (2001), «What Does Ecotourism Means for Europe? » Industry and Environment, Ecotourism, vol. 24, no 3-4, Ecotourism and Sustainability.

Blangy, S. / SECA (2002), Le marché de l'Écotourisme en France - OMT, WTO - World Tourism Organisation Publications.

Blangy, S., J.C. Génot, et M. Criventchuk (1996), « Écotourisme dans la réserve de Bérézinski en Bélarus », Ecodecision, no 20, Canada.

Breton, Jean-Marie (dir.) (2001), L'écotourisme, un nouveau défi pour la Caraïbe ? Éditions Karthala-Crejeta.

Denmann, Richard (2000), Pour un tourisme rural de qualité, La gestion intégrée de la qualité (GIQ) des destinations touristiques rurales, Direction générale des entreprises, Unité « tourisme », Bruxelles, Commission européenne http://europa.eu.int/comm/enterprise/ library/lib-tourism/index.htm.

Dubois, Céron (2002), « Les enjeux oubliés du tourisme durable », Espaces, no 192, avril.

Ecosystems Ltd. (2002), « Sustainable Tourism Based on Natural and Cultural Heritage », Commission européenne, DG Entreprise, Unité tourisme. Bruxelles.

Ecotrans e.V. (2002), Voluntary Initiatives for Sustainable Tourism - Worldwide, Final report for the WTO, Saarbrücken.

GTZ (2002), « Cooperating for Sustainable Tourism », Proceedings of the Forum International at the Reisepavillon 2002, Kasparek Verlag, Hanovre.

OMT - Organisation mondiale du tourisme (2002), Le marché de l'écotourisme en Allemagne, Espagne, France, Italie et Royaume Uni, OMT, WTO - World Tourism Organisation Publications.

Priestley G.K., J.A. Edwards, et H. Coccossis (eds.) (1996), Sustainable Tourism? European Experiences, Cab International, London. 